\title{
Phase transitions in confined water nanofilms
}

\author{
Sungho Han ${ }^{1 \star}$, M. Y. Choi ${ }^{1,2}$, Pradeep Kumar ${ }^{3}$ and H. Eugene Stanley ${ }^{1}$
}

Bulk water has three phases: solid, liquid and vapour. In addition to undergoing a phase transition (of the first order) between them, liquid and vapour can deform continuously into each other without crossing a transition line-in other words, there is no intrinsic distinction between the two phases. Hence, the first-order line of the liquid-vapour phase transition should terminate at a critical point. In contrast, the firstorder transition line between solid and liquid is believed to persist indefinitely without terminating at a critical point ${ }^{1}$. In recent years, however, it was reported that inside carbon nanotubes, freezing of water may occur continuously as well as discontinuously through a first-order phase transition ${ }^{2}$. Here we present simulation results for water in a quasi-twodimensional hydrophobic nanopore slit, which are consistent with the idea that water may freeze by means of both firstorder and continuous phase transitions. Our results lead us to hypothesize the existence of a connection point at which first-order and continuous transition lines meet ${ }^{3,4}$.

It is widely believed that a solid-liquid phase transition line does not terminate at a critical point, although it has not been possible to prove rigorously the non-existence of the solid-liquid critical point ${ }^{1}$. On the other hand, after the discovery of carbon nanotubes ${ }^{5}$, a solid-liquid phase transition inside these structures has been reported, together with the intriguing possibility of a solid-liquid critical point, presumably because of the constraints in packing inside carbon nanotubes ${ }^{6}$. In recent years, therefore, there has been a great deal of interest in the simulation report that freezing of water inside carbon nanotubes may occur continuously as well as discontinuously through a first-order transition ${ }^{2,6,7}$.

As for the solid-liquid phase transition, two-dimensional systems are of particular interest. According to the MerminWagner theorem, continuous symmetry in two dimensions cannot be broken at non-zero temperatures, which means that there is no true long-ranged crystalline order in two-dimensional systems ${ }^{8}$. However, this does not necessarily rule out the presence of a phase transition; instead the Kosterlitz-Thouless-HalperinNelson-Young (KTHNY) theory states that melting in two dimensions may involve two continuous transitions, one from the solid phase to a hexatic phase and the other from the hexatic phase to the liquid phase $\mathrm{e}^{9}$. Although the KTHNY theory seems rather appealing and general, it is still controversial whether the KTHNY theory provides the only mechanism of the melting in two dimensions ${ }^{9,10}$. Here we present simulation results supporting the possibility that a solid-liquid phase transition of water in quasi-twodimensions may occur through both first-order and continuous phase transitions, depending on the density or pressure.

We carry out extensive molecular dynamics simulations of the TIP5P model $^{11}$ of $N=256$ water molecules confined between two unstructured and smooth hydrophobic plates (see ref. 12 for detailed simulation descriptions). We choose the separation $H=0.8 \mathrm{~nm}$ between two parallel $(L \times L)$ plates to contain maximally two layers of water molecules, so that confinement effects on water are extremely strong. We use the linear size of the plate from $L=32.01 \AA$ up to $L=42.70 \AA$, depending on the density $\rho$. The water-wall interactions are modelled by a 9-3 LennardJones potential, which is commonly used to represent the effective interactions of water molecules with the confining surfaces ${ }^{13,14}$. In molecular dynamics simulations we adopt the constant molecule number, volume and temperature (NVT) ensemble with periodic boundary conditions in the parallel ( $x$ and $y$ ) directions, and analyse wide ranges of $\rho$ from $0.81 \mathrm{~g} \mathrm{~cm}^{-3}$ up to $1.45 \mathrm{~g} \mathrm{~cm}^{-3}$ and the temperature $T$ from $300 \mathrm{~K}$ down to $230 \mathrm{~K}$. We carry out molecular dynamics simulations for 10-60 ns, depending on $T$ and $\rho$. To confirm the results of NVT ensemble simulations, we also carry out molecular dynamics simulations on the $N P_{x y} T$ ensemble, where $P_{x y}$ is the pressure in the direction parallel to the plates. We present the results of $N P_{x y} T$ ensemble simulations in the Supplementary Information.

Figure 1 shows the potential energy $U$ as a function of $T$ for various densities. For a range of constant density paths from $\rho=0.99 \mathrm{~g} \mathrm{~cm}^{-3}$ up to $1.28 \mathrm{~g} \mathrm{~cm}^{-3}$, as $T$ decreases, we find large drops in $U\left(5-6 \mathrm{~kJ} \mathrm{~mol}^{-1}\right)$. The temperature range in which the drop occurs depends on the density. Such a sudden drop in $U$ is consistent with the presence of a first-order phase transition between liquid and solid at the temperature $T_{0}$ of the drop ${ }^{15,16}$. For $\rho \geq 1.33 \mathrm{~g} \mathrm{~cm}^{-3}$, on the other hand, the sharp drop suddenly disappears and $U$ decreases gradually as $T$ is lowered to $230 \mathrm{~K}$. It is of interest to compare the two cases, $\rho=1.33 \mathrm{~g} \mathrm{~cm}^{-3}$ and $1.30 \mathrm{~g} \mathrm{~cm}^{-3}$. Whereas the two exhibit the same behaviour at temperatures $T=300 \mathrm{~K}$ down to $260 \mathrm{~K}$, different behaviours begin to develop for $T \leq 250 \mathrm{~K}$. For a continuous drop of $U$ for $\rho \geq 1.33 \mathrm{~g} \mathrm{~cm}^{-3}$, there are three possible explanations. One is that water remains in the liquid state from $T=300 \mathrm{~K}$ down to $230 \mathrm{~K}$, so that there is no sudden drop in $U$. Another is the existence of a weak first-order phase transition between liquid and solid, where the drop is too small to measure in our simulations. The third is the presence of a continuous phase transition at some temperature between $300 \mathrm{~K}$ and $230 \mathrm{~K}$. In the last two cases there would exist a critical density $\rho_{\mathrm{c}}$ between $1.30 \mathrm{~g} \mathrm{~cm}^{-3}$ and $1.33 \mathrm{~g} \mathrm{~cm}^{-3}$, above which the phase transition becomes (quasi-) continuous.

To determine which scenario is correct, we first consider the lateral oxygen-oxygen radial distribution function (RDF) $g_{\mathrm{OO}} x y$ versus the lateral position $r_{x y}$ parallel to the confining plates ${ }^{17}$, and examine explicitly how the structure of water in the parallel direction changes. For $\rho=1.28 \mathrm{~g} \mathrm{~cm}^{-3}$, we observe characteristic features of liquid and of solid in Fig. 2 at high and at low temperatures, respectively. At $T=280 \mathrm{~K}$, the RDF exhibits typical liquid behaviour, which consists of a pre-peak representing a

\footnotetext{
${ }^{1}$ Center for Polymer Studies and Department of Physics, Boston University, Boston, Massachusetts 02215, USA, ${ }^{2}$ Department of Physics and Astronomy, Seoul National University, Seoul 151-747, Republic of Korea, ${ }^{3}$ Center for Studies in Physics and Biology, The Rockefeller University, New York, New York 10021, USA. *e-mail: sungho@bu.edu.
} 
a

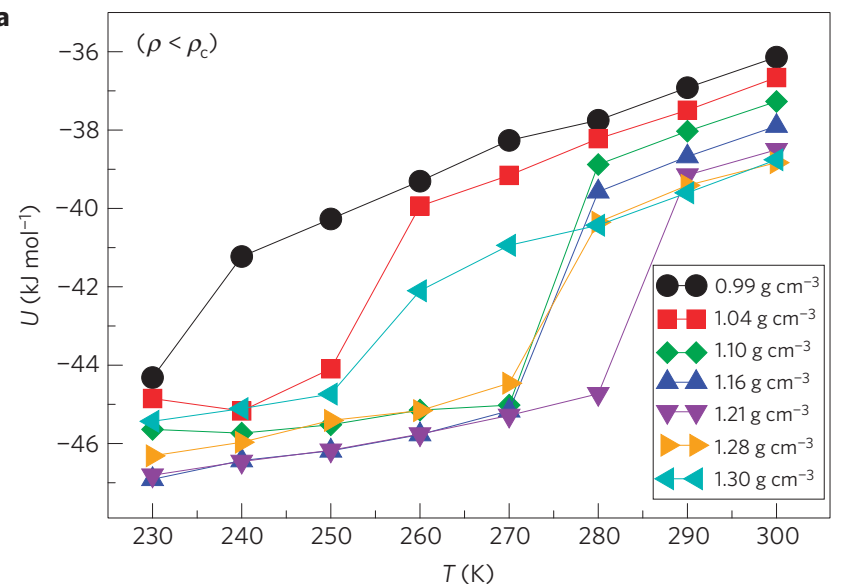

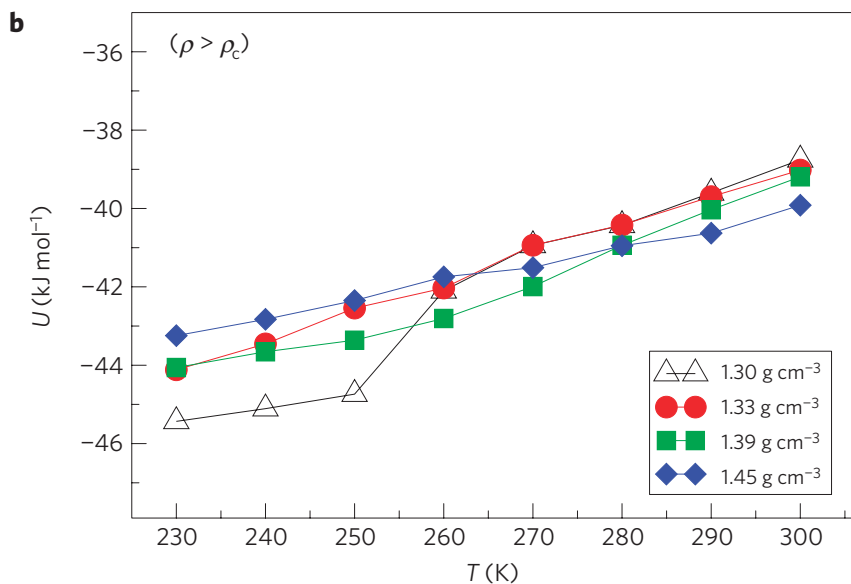

Figure 1 | Discontinuous versus continuous behaviour of potential energy below and above the critical density. a,b, Potential energy $U$ as a function of temperature $T$ for densities $\rho=1.30 \mathrm{~g} \mathrm{~cm}^{-3}$ and below (a) and densities $\rho=1.33 \mathrm{~g} \mathrm{~cm}^{-3}$ and above (b). At density $\rho=1.16 \mathrm{~g} \mathrm{~cm}^{-3}$, on cooling from $T=300 \mathrm{~K}$, there is a sudden drop $\left(\sim 6 \mathrm{~kJ} \mathrm{~mol}^{-1}\right)$ in $U$ across the transition temperature $T_{0}$ between $280 \mathrm{~K}$ and $270 \mathrm{~K}$, which manifests a first-order phase transition between solid and liquid. Such transition behaviour persists up to $\rho=1.30 \mathrm{~g} \mathrm{~cm}^{-3}$, although the drop in $U$ is reduced $\left(\sim 3 \mathrm{~kJ} \mathrm{~mol}{ }^{-1}\right)$ with its location $T_{0}$ depending on the density. When $\rho$ reaches $1.33 \mathrm{~g} \mathrm{~cm}^{-3}$, however, there is no sharp drop and $U$ decreases gradually as $T$ is lowered down to $230 \mathrm{~K}$. We thus suggest that as the density is raised, the nature of the phase transition might change from a first-order transition to a continuous one at the critical density $\rho_{\mathrm{c}}$, located between $1.30 \mathrm{~g} \mathrm{~cm}^{-3}$ and $1.33 \mathrm{~g} \mathrm{~cm}^{-3}$.

a

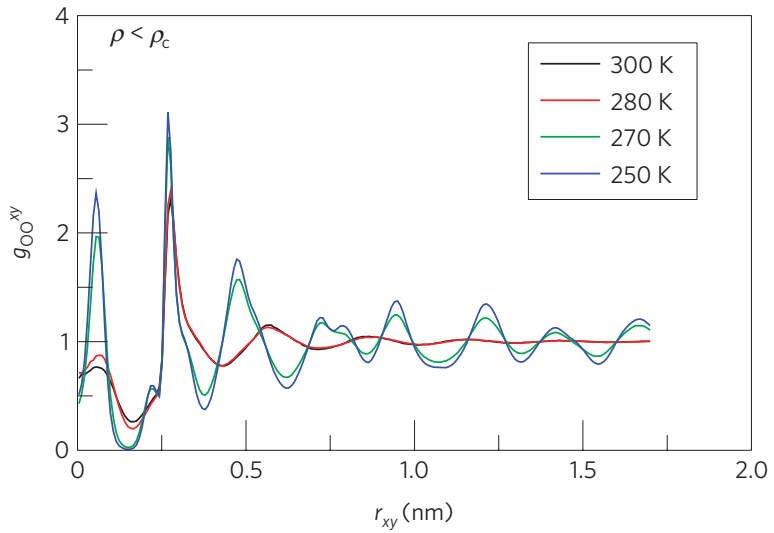

c

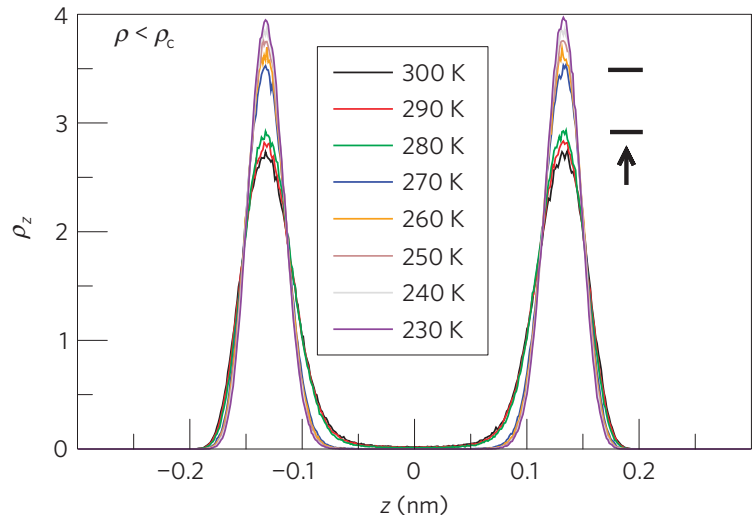

b

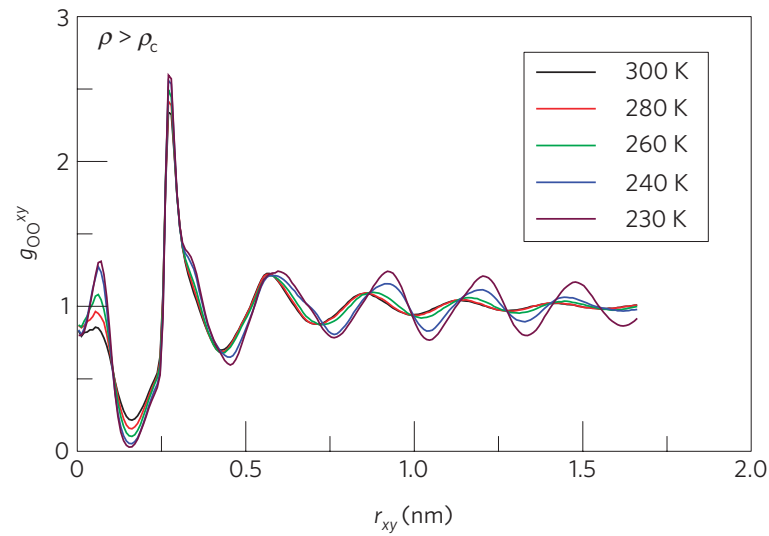

d

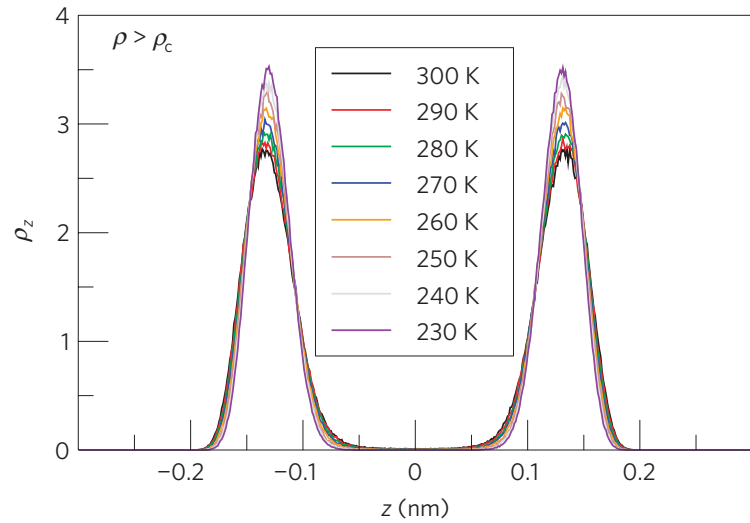

Figure 2 | Lateral oxygen-oxygen RDF $g_{00}{ }^{x y}$ and TDP $\rho_{\mathbf{z}}$ at various temperatures. $\mathbf{a}$, The RDF at density $\rho=1.28 \mathrm{~g} \mathrm{~cm}{ }^{-3}\left(<\rho_{\mathrm{c}}\right)$ exhibits characteristic features of liquids and of solids, respectively, depending on temperature $T$. When $T$ is varied across $270 \mathrm{~K}$ and $280 \mathrm{~K}$, the RDF changes abruptly, indicating a first-order transition between liquid and solid. $\mathbf{b}$, The RDF at $\rho=1.33 \mathrm{~g} \mathrm{~cm}^{-3}\left(>\rho_{\mathrm{c}}\right)$, unlike that for $\rho<\rho_{\mathrm{c}}$, varies continuously rather than abruptly over the entire temperature range. At $T=230 \mathrm{~K}$, it exhibits long-range order, corresponding to the crystalline order. As $T$ is raised, the long-range order of the RDF becomes faint, turning into liquid-like disorder. $\mathbf{c}$, The TDP at $\rho=1.28 \mathrm{~g} \mathrm{~cm}^{-3}$ also exhibits the abrupt change of the structure seen for the RDF at the same temperature between $270 \mathrm{~K}$ and $280 \mathrm{~K}$. This indicates that the structural change occurs discontinuously in the direction both parallel and perpendicular to the confining surfaces. $\mathbf{d}$, At $\rho=1.33 \mathrm{~g} \mathrm{~cm}^{-3}$, the TDP varies gradually with $T$, which is again consistent with the structural change observed in the RDF. Accordingly, behaviours of both the RDF and the TDP support the scenario that the phase transition between liquid and solid changes from a first-order transition to a continuous one, as $\rho$ is increased above $\rho_{\mathrm{c}}$. 

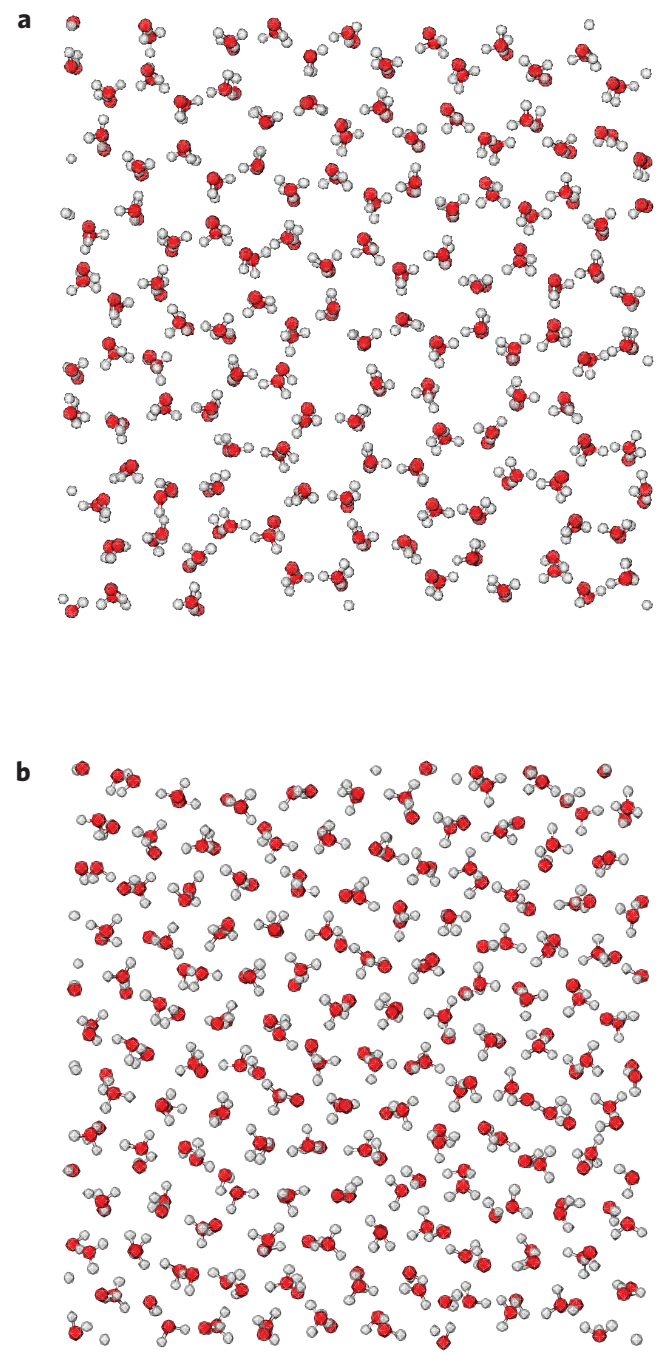

Figure 3 | Two different ice structures below and above the critical density. a,b, Snapshots of the top view of two different phases of solid water at $T=240 \mathrm{~K}$ with $\rho<\rho_{\mathrm{c}}(\mathbf{a})$ and $\rho>\rho_{\mathrm{c}}(\mathbf{b})$. The formation of ice for $\rho<\rho_{\mathrm{c}}$ is hexagonal, whereas it is rhombic for $\rho>\rho_{\mathrm{c}}$.

bilayer, and the prominent first peak at $r_{x y}=0.28 \mathrm{~nm}$, followed by rapidly decaying peaks and the eventual approach to unity indicating the disordered state ${ }^{12}$. At $T=270 \mathrm{~K}$, on the other hand, the RDF exhibits long-ranged order, showing that water has transformed to ice. The behaviour of the RDF, characteristic of a first-order transition ${ }^{1}$, changes abruptly when the temperature is varied across the transition temperature $T_{0}$ between $270 \mathrm{~K}$ and $280 \mathrm{~K}$. For the higher density $\rho=1.33 \mathrm{~g} \mathrm{~cm}^{-3}$, the RDF at low temperatures also shows long-ranged order, again corresponding to ice. However, the temperature dependence of the RDF exhibits behaviour different from that in the case of lower densities. Instead of the abrupt disappearance of the long-ranged order observed for $\rho=1.28 \mathrm{~g} \mathrm{~cm}^{-3}$, the long-ranged order gradually reduces as the temperature is raised. Such gradual (rather than abrupt) variation of the RDF with temperature, which persists at higher densities $\left(\rho \geq 1.33 \mathrm{~g} \mathrm{~cm}^{-3}>\rho_{\mathrm{c}}\right)$, illustrates that the phase changes continuously, in contrast with the case of lower densities $\left(\rho \leq 1.30 \mathrm{~g} \mathrm{~cm}^{-3}<\rho_{\mathrm{c}}\right.$ ) exhibiting a discontinuous phase change. Therefore, the RDF results suggest that the nature of the phase transition between solid and liquid changes across the critical density $\rho=\rho_{\mathrm{c}}$, where $1.30 \mathrm{~g} \mathrm{~cm}^{-3}<\rho_{\mathrm{c}}<1.33 \mathrm{~g} \mathrm{~cm}^{-3}$. Note that ice formations for $\rho<\rho_{\mathrm{c}}$ and $\rho>\rho_{\mathrm{c}}$ are different. For $\rho>\rho_{\mathrm{c}}$ ice structure is rhombic, whereas for $\rho<\rho_{\mathrm{c}}$ it is hexagonal (Fig. 3).

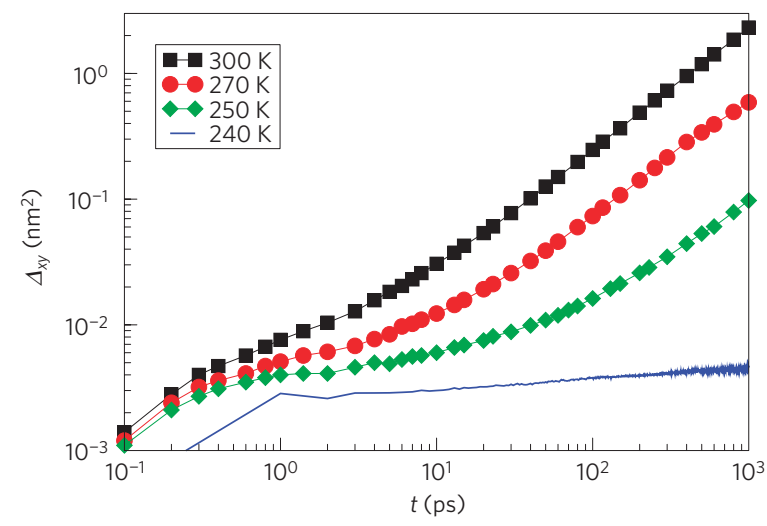

Figure 4 | Log-log plot of the time evolution of the lateral MSD $\Delta_{x y}$ at several temperatures and density $\rho=1.33 \mathrm{~g} \mathrm{~cm}^{-3}\left(>\rho_{\mathrm{c}}\right)$.

At temperatures $T=300 \mathrm{~K}$ down to $250 \mathrm{~K}$, the MSD, starting from the ballistic regime $\left(\alpha t^{2}\right)$, eventually reaches the diffusive regime $(\alpha t)$ in the long time $t$. The plateau between the two regimes, observed at low temperatures, arises from the cage effect. We thus conclude that the MSD exhibits typical liquid behaviour. At $T=240 \mathrm{~K}$, in contrast, the evolution of the MSD is almost flat and exhibits large fluctuations, indicating that water has turned into ice. The diffusion constant $D$, calculated from the MSD, takes the values $5.7 \times 10^{-6} \mathrm{~cm}^{2} \mathrm{~s}^{-1}, 1.3 \times 10^{-6} \mathrm{~cm}^{2} \mathrm{~s}^{-1}$ and $2.2 \times 10^{-7} \mathrm{~cm}^{2} \mathrm{~s}^{-1}$ at $T=300 \mathrm{~K}, 270 \mathrm{~K}$ and $250 \mathrm{~K}$, respectively, whereas at $T=240 \mathrm{~K}$ it is given by $5.2 \times 10^{-9} \mathrm{~cm}^{2} \mathrm{~s}^{-1}$.

During slow cooling and heating processes, we also find that $U$ shows a hysteresis loop for $\rho<\rho_{\mathrm{c}}$ but rather a continuous change without a hysteresis loop for $\rho>\rho_{\mathrm{c}}$, supporting two different phase transitions (see the Supplementary Information).

To confirm the presence of two different solid-liquid phase transitions, we next examine the structural change of water in the transverse (confining) direction. The difference in the structural behaviour for $\rho<\rho_{\mathrm{c}}$ and for $\rho>\rho_{\mathrm{c}}$ is revealed in the transverse density profile (TDP) $\rho_{z}$ versus the confining coordinate $z$ (ref. 17). In Fig. 2, the TDP for $\rho=1.28 \mathrm{~g} \mathrm{~cm}^{-3}\left(<\rho_{\mathrm{c}}\right)$ exhibits big jumps in the two peaks at $T \approx T_{0}$ between $270 \mathrm{~K}$ and $280 \mathrm{~K}$. Namely, the structure in the direction perpendicular to the confining surfaces also changes discontinuously at $T=T_{0}$. For $\rho=1.33 \mathrm{~g} \mathrm{~cm}^{-3}\left(>\rho_{\mathrm{c}}\right)$, on the other hand, the two peaks of the TDP do not show any appreciable jumps with temperature; instead the TDP varies gradually with $T$, similar to the RDF for $\rho>\rho_{\mathrm{c}}$. These consistent behaviours of the RDF and the TDP support our hypothesis that the nature of the phase transition between solid and liquid changes as $\rho$ is varied across $\rho_{c}$. From the $N P_{x y} T$ simulations for $P_{x y}<P_{c}$ and $P_{x y}>P_{\mathrm{c}}$ (where $P_{\mathrm{c}}$ is the critical lateral pressure), we find that $\rho$ changes discontinuously by crossing a transition line for $P_{x y}<P_{\mathrm{c}}$. In contrast, it changes continuously for $P_{x y}>P_{c}$, manifesting two different phase transitions (see the Supplementary Information).

We further probe the dynamical properties for $\rho>\rho_{\mathrm{c}}$, which are conveniently characterized by the lateral mean-square displacement (MSD) $\Delta_{x y}(t) \equiv\left\langle\left[r_{x y}(t)-r_{x y}(0)\right]^{2}\right\rangle$ (ref. 17). Figure 4 shows the mobility difference between liquid and solid, measured by the MSD as a function of time $t$, for density $\rho=1.33 \mathrm{~g} \mathrm{~cm}^{-3}$ at various temperatures. At $T=300 \mathrm{~K}$, the MSD, starting initially from the ballistic regime $\left(\propto t^{2}\right)$, finally reaches the diffusive regime $(\propto t)$. This behaviour persists down to $T=250 \mathrm{~K}$, which suggests a liquid phase, although the slope of the MSD continues to decrease as $T$ is lowered. At $T=240 \mathrm{~K}$, the MSD remains almost flat (constant) with time, indicating that water has frozen to ice. Although the mobility at temperatures from $300 \mathrm{~K}$ down to $250 \mathrm{~K}$ is distinguishable from that at $T=240 \mathrm{~K}$, it varies in a continuous way; the slope of the MSD for the liquid approaches continuously the slope for the solid. Such continuity is not observed in the case of a first-order 


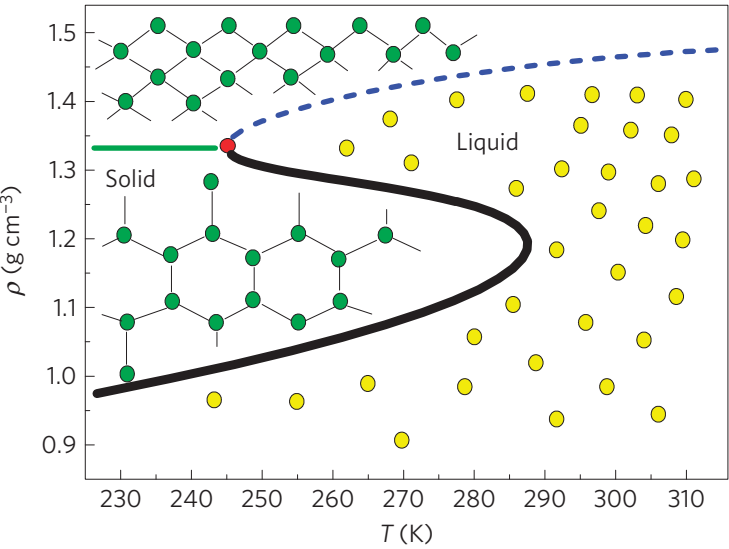

Figure 5 | Schematic phase diagram of confined water in the density-temperature $(\rho-T)$ plane, estimated from behaviours of the potential energy, RDF, TDP and MSD. Whereas at low densities confined water undergoes a phase transition between solid (green circles) and liquid (yellow circles) phases by crossing a first-order line, the phase changes rather continuously for $\rho$ exceeding $\rho_{\mathrm{c}}$. Accordingly, the first-order transition line (represented by the black solid line) continues to a continuous transition line (the blue dashed line), and there is a connection point between two transition lines (the red bullet). The connection point is located in the ranges of density $1.30 \mathrm{~g} \mathrm{~cm}^{-3}<\rho_{\mathrm{c}}<1.33 \mathrm{~g} \mathrm{~cm}^{-3}$ and of temperature $240 \mathrm{~K}<T_{\mathrm{c}}<250 \mathrm{~K}$, whereas the location of a transition line has an uncertainty $\Delta T \sim \pm 5 \mathrm{~K}$ and $\Delta \rho \sim \pm 0.05 \mathrm{~g} \mathrm{~cm}^{-3}$. In the $\rho-T$ phase diagram, a first-order phase transition should be represented by a region of the two-phase coexistence. However, the finite size of the system and the choice of the NVT ensemble apparently lead the coexistence region to be narrow, so that the coexistence region here appears as a line. Here, $\rho$ in the NVT ensemble plays almost the same role as $P$ in the NPT ensemble. Two ice phases have different symmetries and are thus separated by a phase boundary (denoted by a green solid line).

transition. It has been shown that the diffusion constant $D$, which is related to the slope of MSD through Einstein's relation ${ }^{17}$, changes abruptly when the first-order transition line is crossed $^{18}$. For ice, $D \sim 10^{-10} \mathrm{~cm}^{2} \mathrm{~s}^{-1}$, whereas for liquid water $D \sim 10^{-5} \mathrm{~cm}^{2} \mathrm{~s}^{-1}$ (refs $2,12,16,18,19)$. Therefore, the mobility can serve as a good measure of a phase transition ${ }^{20}$ and provides further evidence for the existence of both the solid and the liquid phases separated by a (continuous) phase transition for $\rho>\rho_{\mathrm{c}}$. Such continuous freezing has been experimentally observed in diffraction measurements of water inside cylindrical nanopores ${ }^{21}$.

In addition to the well-known liquid-vapour critical point, another critical point of the liquid-liquid phase transition has been found in simulations of water ${ }^{22}$. As for phases of solid and liquid, however, a symmetry argument forbids the presence of a solidliquid critical point in bulk water ${ }^{1}$. For confined water, noteworthy in the limit of high density are the layering effects, which arise from confining boundary conditions ${ }^{17,23}$. Layering, observed in the TDP for high densities, in general hinders water molecules from leaving the layer and facilitates them to form a relatively ordered structure compared with typical liquid water. As $T$ is subsequently lowered, water is expected to freeze without changing its relatively ordered structure, so the structure of liquid water should be similar to that of ice $^{2}$. As a quasi-two-dimensional system, monolayer water may not be expected to show a continuous transition at high pressures, because the freezing of monolayer water at high pressures is generally coupled to a first-order buckling transition ${ }^{12,18}$.

In the case of water in a quasi-two-dimensional hydrophobic nanopore slit, we have found that the first-order transition line may connect with a continuous transition line (see Fig. 5), instead of terminating at the critical point. The resulting connection point between the two transition lines is located at $1.30 \mathrm{~g} \mathrm{~cm}^{-3}<\rho_{\mathrm{c}}<$ $1.33 \mathrm{~g} \mathrm{~cm}^{-3}$ and $240 \mathrm{~K}<T_{\mathrm{c}}<250 \mathrm{~K}$. This is in contrast to the quasi-one-dimensional system of water inside carbon nanotubes, where the first-order transition line terminates at a solid-liquid critical point ${ }^{2}$, and beyond the point it seems that one phase can deform continuously to the other without crossing a transition line, similarly to the case of a liquid-vapour critical point ${ }^{1,2}$. Even in three dimensions there is a simulation result reporting evidence for a solid-liquid critical point in a simple monatomic system $^{24}$. Recall that the mobility of water molecules presented in Fig. 4 seems supportive of the existence of a continuous phase transition at high densities. Such a continuous transition was also observed between the liquid and the hexatic phase in a monolayer of Lennard-Jones fluids inside a quasi-two-dimensional pore $\operatorname{slit}^{25}$. In addition, simulations of a two-dimensional system with short-range attractive interactions revealed a solid-solid critical point that terminates a first-order transition line between two solids ${ }^{26}$.

Whereas for liquid water unstructured walls in general reproduce the same results as atomic and structured walls ${ }^{27}$, this is not clear for a solid-liquid phase transition. In view of the fact that the phase transition in confined water is surface-induced ${ }^{28}$, it is likely that the nature of confining walls should affect the freezing mechanism of water. In particular, in the search for a continuous solid-liquid phase transition, hydrophobicity of surfaces might be crucial, as both in experiments ${ }^{29}$ and in simulations ${ }^{16}$ freezing has not been observed for water confined in hydrophilic environments. Although our finding is consistent with a number of existing results in (quasi-)two dimensions, we do not exclude the possibility of firstorder phase transitions at high densities rounded off by finite-size effects. Concerning experimental evidence, the recent report of ice at room temperature formed from nanoconfined water ${ }^{30}$, as well as the measurement of water inside nanopores ${ }^{21}$, is encouraging.

\section{Received 5 June 2009; accepted 21 May 2010; published online} 4 July 2010

\section{References}

1. Stanley, H. E. Introduction to Phase Transitions and Critical Phenomena (Oxford Univ. Press, 1971).

2. Koga, K., Gao, G. T., Tanaka, H. \& Zeng, X. C. Formation of ordered ice nanotubes inside carbon nanotubes. Nature 412, 802-805 (2001).

3. Landau, L. D. \& Lifshitz, E. M. Statistical Physics: Part 1 (Butterworth-Heinemann, 2001).

4. Griffiths, R. B. Thermodynamics near the two-fluid critical mixing point in $\mathrm{He}^{3}-\mathrm{He}^{4}$. Phys. Rev. Lett. 24, 715-717 (1970).

5. Iijima, S. Helical microtubules of graphitic carbon. Nature 354, 56-58 (1991).

6. Ball, P. New horizons in inner space. Nature 361, 297 (1993).

7. Koga, K. Freezing in one-dimensional liquids. J. Chem. Phys. 118, 7973-7980 (2003).

8. Mermin, N. D. Crystalline order in two dimensions. Phys. Rev. 176, 250-254 (1968)

9. Strandburg, K. J. Two-dimensional melting. Rev. Mod. Phys. 60, 161-207 (1988).

10. Dash, J. G. History of the search for continuous melting. Rev. Mod. Phys. 71, 1737-1743 (1999).

11. Mahoney, M. W. \& Jorgensen, W. L. A five-site model for liquid water and the reproduction of the density anomaly by rigid, nonpolarizable potential functions. J. Chem. Phys. 112, 8910-8922 (2000).

12. Kumar, P., Buldyrev, S. V., Starr, F. W., Giovambattista, N. \& Stanley, H. E. Thermodynamics, structure, and dynamics of water confined between hydrophobic plates. Phys. Rev. E 72, 051503 (2005).

13. Steele, W. A. The Interaction of Gases with Solid Surfaces (Oxford, 1974).

14. Han, S., Kumar, P. \& Stanley, H. E. Hydrogen-bond dynamics of water in a quasi-two-dimensional hydrophobic nanopore slit. Phys. Rev. E 79, 041202 (2009).

15. Koga, K., Zeng, X. C. \& Tanaka, H. Freezing of confined water: A bilayer ice phase in hydrophobic nanopores. Phys. Rev. Lett. 79, 5262-5265 (1997).

16. Koga, K., Tanaka, H. \& Zeng, X. C. First-order transition in confined water between high-density liquid and low-density amorphous phases. Nature 408, 564-567 (2000).

17. Hansen, J-P. \& McDonald, I. R. Theory of Simple Liquids (Academic, 2006).

18. Zangi, R. Water confined to a slab geometry: A review of recent computer simulation studies. J. Phys. Condens. Matter 16, S5371-S5388 (2004). 
19. Han, S., Kumar, P. \& Stanley, H. E. Absence of a diffusion anomaly of water in the direction perpendicular to hydrophobic nanoconfining walls. Phys. Rev. E 77, 030201(R) (2008).

20. Hedges, L. O., Jack, R. L., Garrahan, J. P. \& Chandler, D. Dynamic order-disorder in atomistic models of structural glass formers. Science 323, 1309-1313 (2009).

21. Morishige, K. \& Kawano, K. Freezing and melting of water in a single cylindrical pore: The pore-size dependence of freezing and melting behaviour. J. Chem. Phys. 110, 4867-4872 (1999).

22. Poole, P. H., Sciortino, F., Essmann, U. \& Stanley, H. E. Phase behaviour of metastable water. Nature 360, 324-328 (1992).

23. Gao, J., Luedtke, W. D. \& Landman, U. Layering transitions and dynamics of confined liquid films. Phys. Rev. Lett. 79, 705-708 (1997).

24. Elenius, M. \& Dzugutov, M. Evidence for a liquid-solid critical point in a simple monatomic system. J. Chem. Phys. 131, 104502 (2009).

25. Radhakrishnan, R., Gubbins, K. E. \& Sliwinska-Bartkowiak, M. Existence of a hexatic phase in porous media. Phys. Rev. Lett. 89, 076101 (2002).

26. Bladon, P. \& Frenkel, D. Dislocation unbinding in dense two-dimensional crystals. Phys. Rev. Lett. 74, 2519-2522 (1995).

27. Lee, S. H. \& Rossky, P. J. A comparison of the structure and dynamics of liquid water at hydrophobic and hydrophilic surfaces-a molecular dynamics simulation study. J. Chem. Phys. 100, 3334-3345 (1994).
28. Giovambattista, N., Rossky, P. J. \& Debenedetti, P. G. Phase transitions induced by nanoconfinement in liquid water. Phys. Rev. Lett. 102, 050603 (2009).

29. Raviv, U., Laurat, P. \& Klein, P. J. Fluidity of water confined to subnanometre films. Nature 413, 51-54 (2001).

30. Jinesh, K. B. \& Frenken, J. W. M. Experimental evidence for ice formation at room temperature. Phys. Rev. Lett. 101, 036101 (2008).

\section{Acknowledgements}

We thank S. V. Buldyrev for helpful discussions. S.H., P.K. and H.E.S. acknowledge support from NSF Grant No. CHE 0606489 and the Keck Foundation NAKFI award. M.Y.C. acknowledges support from NRF through the Basic Science Research Program (2009-0080791).

\section{Author contributions}

S.H., M.Y.C., P.K. and H.E.S. designed the research; S.H. carried out simulations; and S.H., M.Y.C., P.K. and H.E.S. analysed data and wrote the paper.

\section{Additional information}

The authors declare no competing financial interests. Supplementary information accompanies this paper on www.nature.com/naturephysics. Reprints and permissions information is available online at http://npg.nature.com/reprintsandpermissions. Correspondence and requests for materials should be addressed to S.H. 